\title{
Mucinous adenocarcinoma of the bladder: A case report and review of the literature
}

\author{
XIANG PAN $^{1,2^{*}}$, LU JIN $^{1,2^{*}}$, TAO HE ${ }^{2,3}$, JIA HU $^{2,3}$, JING QUAN $^{1,2}$, LIJUN ZHOU $^{2}$, \\ LIANGCHAO NI $^{2}$, SHANGQI YANG ${ }^{2}$, XIANGMING MAO $^{2}$ and YONGQING LAI ${ }^{2}$ \\ ${ }^{1}$ Department of Clinical Medicine, The First Clinical College, Anhui Medical University, Hefei, \\ Anhui 230032; ${ }^{2}$ Department of Urology, Peking University Shenzhen Hospital, Shenzhen, \\ Guangdong 518036; ${ }^{3}$ Guangzhou Medical University, Guangzhou, Guangdong 510006, P.R. China
}

Received May 4, 2016; Accepted July 4, 2016

DOI: $10.3892 / \mathrm{mco} .2016 .985$

\begin{abstract}
Mucinous adenocarcinoma of the bladder is rare. The most common symptoms are hematuria, suprapubic pain and dysuria. The patient in the present case was a 59-year-old female who presented with a tumor sized $\sim 5.0 \times 3.4 \mathrm{~cm}$, located in the anterior bladder wall, which was pathologically diagnosed as mucinous bladder adenocarcinoma. The stage at presentation was considered to be advanced and inoperable, due to extensive local invasion. For this type of cancer, early diagnosis is crucial. If the cancer is limited to the bladder, the survival rate may exceed $75 \%$. Thus, for patients with confirmed or highly suspicious bladder mucinous adenocarcinoma, radical bladder cancer resection is required. The 5-year survival rate of this type of cancer is $35-55 \%$. In the present case, the cancer of the patient was considered inoperable and 3 months later she succumbed to the disease.
\end{abstract}

\section{Introduction}

Bladder cancer is a very common cancer of the urinary system and the ninth most common type of cancer worldwide, with a reported 386,000 new cases leading to 150,000 deaths worldwide in 2014 (1). The majority of bladder cancer cases (90-95\%) are urothelial carcinomas, with squamous cell carcinoma and adenocarcinoma representing only 3 and $2 \%$ of the cases, respectively $(2,3)$. The mucinous adenocarcinoma subtype is extremely rare (4). According to a previous study, there is a progressive change from mucinous metaplasia to mucinous adenoma to mucinous adenocarcinoma (5). Patients with bladder exstrophy and urachal remnants are at higher

Correspondence to: Professor Yongqing Lai, Department of Urology, Peking University Shenzhen Hospital, 1120 Lianhua Road, Shenzhen, Guangdong 518036, P.R. China

E-mail: yqlord@163.com

${ }^{*}$ Contributed equally

Key words: bladder tumor, mucinous adenocarcinoma of the bladder, adenocarcinoma risk of developing bladder adenocarcinoma (6). It was also suggested that chronic inflammatory processes of the bladder mucosa induce metaplastic changes to the more protective squamous cell or glandular-type epithelium (7).

Bladder adenocarcinoma is resistant to chemotherapy and radiation, and surgery is currently considered the most effective treatment option (8). Thus, early diagnosis is crucial; however, early diagnosis may be difficult, as the clinical characteristics of bladder mucinous adenocarcinoma are similar to those of other bladder cancers. The most common symptoms are hematuria, suprapubic pain, and dysuria (9). The most effective diagnostic investigations are urinary cytology, cystoscopy, and biopsy followed by histopathological evaluation. Due to the rarity of this tumor, and the relative lack of clinical reports, we herein report the case of a patient with mucinous adenocarinoma of the bladder to help elucidate the characteristics of this tumor.

\section{Case report}

The patient was a 59-year-old female, menopausal, non-smoker and non-drinker, who presented with epigastric pain for 5 months. The computed tomography (CT) scan of the abdomen revealed a mass located in the anterior bladder wall, sized $5.0 \times 3.4 \mathrm{~cm}$. The surrounding bladder wall was thickened, with no abnormal findings in the pelvic cavity (Fig. 1). Transurethral resection of the bladder tumor with electrocautery was undertaken to investigate the tumor. The pathological results revealed the presence of a significant amount of mucus, with irregular cell cords surrounding the mucus pools. Therefore, the suspected diagnosis was mucinous bladder adenocarcinoma (Fig. 2).

Radical resection and bilateral ureterostomy was planned; however, when the abdominal cavity was opened, the tumor was found to have invaded the greater omentum and peritoneum. Therefore, the cancer of the patient was considered as advanced, with significant local invasion and, thus, inoperable. Three months later, the patient succumbed to the disease.

\section{Discussion}

Mucinous adenocarcinoma of the bladder is a rare type of cancer, accounting for $<2 \%$ of all bladder cancers (10). It usually 


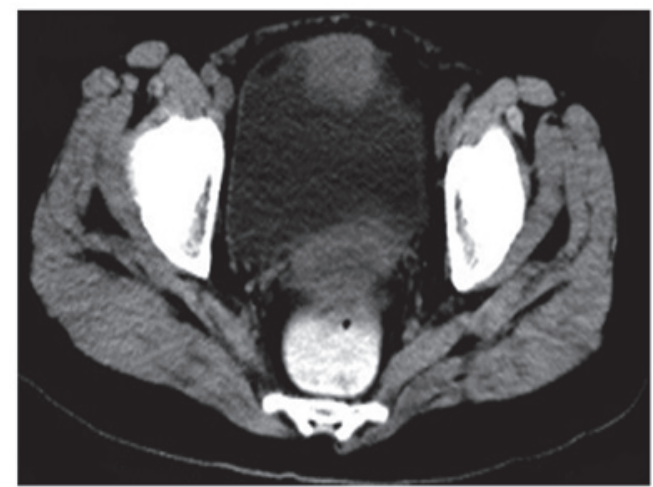

Figure 1. A mass located in the anterior bladder wall was identified on computed tomography, sized $5.0 \times 3.4 \mathrm{~cm}$. The surrounding bladder wall was thickened. There were no abnormal findings in the pelvic cavity.

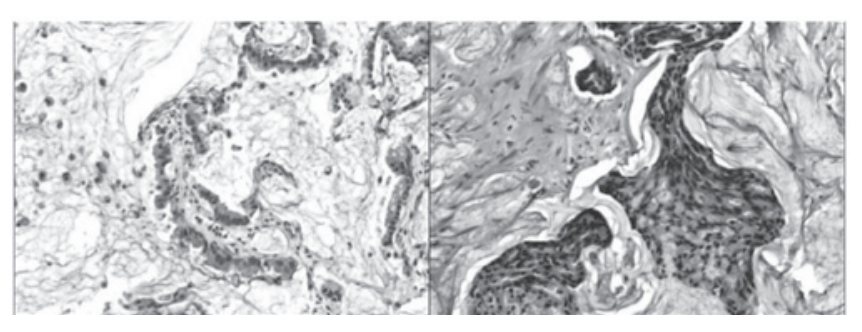

Figure 2. On histological examination, the tumor contained copious amounts of mucus, with irregular cell cords surrounding the mucus pools.

appears at the bladder dome, trigone and lateral wall. There are three hypotheses regarding the histological origin: The first is urachal remnants in the bladder; the second is a vestigial embryonal gland in the transitional epithelium of the bladder; and the third is the transitional epithelium of the bladder undergoing glandular metaplasia. Glandular cystitis is widely considered as a precancerous condition for bladder adenocarcinoma. The mucosa in bladder adenocarcinoma is not smooth, resembling edematous surface villi, or even papilloma-like changes on cystoscopy. The accumulation of secretions and associated infection may promote the development of adenocarcinoma.

As the adenocarcinoma cells secrete copious amounts of mucus, mucous floccules may be discharged from the bladder during micturition or cystoscopy. The main primary symptom is hematuria, with or without signs of irritation of the bladder. A proportion of the patients may only present with signs of bladder irritation and difficulty urinating.

Distinguishing between mucinous bladder adenocarcinoma and urachal carcinoma is crucial, but may be challenging, as their presentations may be similar. The primary clinical signs of bladder adenocarcinoma are hematuria and dysuria, whereas mucusuria is observed in $\sim 90 \%$ of urachal carcinomas. Furthermore, the treatment of urachal carcinoma is partial cystectomy with en bloc resection of the urachus, rather than radical cystectomy. In addition, urachal carcinoma has a better prognosis and high survival rate compared with mucinous adenocarcinoma of the bladder (11).

The prognostic factors of the cancer include tumor stage, grade and subtype. According to a previous study, if the cancer is limited to the bladder, the survival rate may exceed $75 \%$ (6). At this stage, the ultrasound appearance may resemble urinary tract infection (6). Grignon et al identified 5 ubtypes of bladder adenocarcinoma: i) Papillary; ii) mucinous; iii) signet-ring cell; iv) adenocarcinoma not otherwise specified; and v) mixed. The signet-ring cell type has a poorer prognosis compared with that of the other types of adenocarcinoma (12).

The growth pattern of adenocarcinoma cells mainly involves infiltration into the deep muscular layer; thus, cystoscopy and B-mode ultrasound may be unable to assess the extent of infiltration. Therefore, the majority of bladder mucinous adenocarcinoma patients are stage $\mathrm{T} 2$ or $\mathrm{T} 3$ at diagnosis.

When a tumor is identified at the bladder dome, trigone or lateral wall, or in patients presenting with mucous floccules discharged from the bladder during micturition or cystoscopy, the presence of bladder adenocarcinoma should be taken into consideration. Tumor tissue biopsy and CT are required. Several random biopsy samples and regular follow-up visits are also required for patients with glandular cystitis. Bladder adenocarcinoma is resistant to chemotherapy and radiation; thus, for patients with confirmed or highly suspicious mucinous adenocarcinoma of the bladder, timely radical resection is warranted.

\section{Acknowledgements}

The present study was supported by grants from the National Natural Science Foundation of China(no.81101922), the Science and Technology Development Fund Project of Shenzhen (nos. JCY20130402114702124 and JCY20150403091443329) and funds from the Guangdong Key Medical Subject.

\section{References}

1. Chavan S, Bray F, Lortet-Tieulent J, Goodman M and Jemal A: International variations in bladder cancer incidence and mortality. Eu Urol 66: 59-73, 2014.

2. Black PC, Brown GA and Dinney CP: The impact of variant histology on the outcome of bladder cancer treated with curative intent. Urol Oncol 27: 3-7, 2009.

3. Kantor AF, Hartge P, Hoover RN and Fraumeni JF Jr: Epidemiological characteristics of squamous cell carcinoma and adenocarcinoma of the bladder. Cancer Res 48: 3853-3855, 1988.

4. Sigalas K, Tyritzis SI, Trigka E, Katafigiotis I, Kavantzas N and Stravodimos KG: A male presenting with a primary mucinous bladder carcinoma: A case report. Cases J 3: 49, 2010.

5. Zhang BY, Aguilar J, Yang M, Wang P and Li B: Mucinous metaplasia in urothelial tract may be the precancerous lesion of mucinous adenocarcinoma: Report of two cases and review of literature. Int J Clin Exp Med 7: 285-289, 2014.

6. Santos BM, de Souza JD, Lima RS and de Lima EM: Mucinous bladder adenocarcinoma: Case report and literature review. Case Rep Urol 2015: 783109, 2015.

7. Bullock PS, Thoni DE and Murphy WM: The significance of colonic mucosa (intestinal metaplasia) involving the urinary tract. Cancer 59: 2086-2090, 1987.

8. Baffigo G, Delicato G, Bianchi D, Signore S, Tartaglia E, Corvese F, Perla A, Marino B, Santeusanio G and Ferdinandi V: Mucinous adenocarcinoma of the urinary bladder. Am J Case Rep 13: 99-101, 2012.

9. Zaghloul MS, Nouh A, Nazmy M, Ramzy S, Zaghloul AS, Sedira MA and Khalil E: Long-term results of primary adenocarcinoma of the urinary bladder: A report on 192 patients. Urol Oncol 24, 13-20, 2006.

10. Bates AW and Baithun SI: Secondary neoplasms of the bladder are histological mimics of nontransitional cell primary tumours: Clinicopathological and histological features of 282 cases. Histopathology 36: 32-40, 2000.

11. Wright JL, Porter MP, Li CI, Lange PH and Lin DW: Differences in survival among patients with urachal and nonurachal adenocarcinomas of the bladder. Cancer 107: 721-728, 2006.

12. Fiter L, Gimeno F, Martin L and Gómez Tejeda L: Signet-ring cell adenocarcinoma of bladder. Urology 41: 30-33, 1993. 